\title{
Comportement de vitroplants de bananiers plantains issus de bourgeons axillaires et apicaux au cours de l'acclimatation et en champ
}

Emmanuel YoumBI $^{a *}$, Nanga Jean Philipps FonKAM ${ }^{b}$, Dieudonné NGAHA ${ }^{a}$, Michel Ndoumbé NKENG ${ }^{c}$, Moïse KWA ${ }^{d}$

a Centre Africain de

Recherches sur Bananiers et Plantains (CARBAP),

Laboratoire de culture in vitro, BP 832, Douala, Cameroun

youmbi_emmanuel@yahoo.fr

b Université de Yaoundé I, Faculté des Sciences, Laboratoire de physiologie et stress, BP 812, Yaoundé, Cameroun

${ }^{\mathrm{C}}$ IRAD Nkolbisson, BP 2123, Yaoundé, Cameroun

${ }^{d}$ Centre Africain de

Recherches sur Bananiers et Plantains (CARBAP),

Laboratoire d'agronomie, BP 832, Douala, Cameroun

* Correspondance et tirés à part

Reçu le 19 mai 2004

Accepté le 12 janvier 2005

Fruits, 2005, vol. 60, p. 91-100 (C) 2005 Cirad/EDP Sciences All rights reserved

DOI: $10.1051 /$ fruits:2005019

RESUMEN ESPAÑOL, p. 100

\section{Behavior of plantain banana vitroplants resulting from axillary buds and shoot tips during the acclimatization phase and in the field.}

Abstract - Introduction. Plantain banana contributes to the food safety and incomes of subSaharan Africa's inhabitants. The use of healthy plants in banana plantations is an important factor for their establishment. Different methods have been developed to produce plant material free of disease, such as in vivo and in vitro multiplication. In vitro micropropagation by budding allows the production of many plants free of disease. The in vitro multiplication of banana usually takes only one shoot tip $\left(\mathrm{sh}_{\mathrm{t}}\right)$ per sucker. However, a sucker contains many axillary buds $\left(\mathrm{ax}_{\mathrm{b}}\right)$, whose potentialities we evaluated. Materials and methods. Our experiments related to the cultivar Big Ebanga (AAB, plantain false horn). During acclimatization under shade and in the field, we compared growth and development parameters of vitroplants resulting from the two types of explants, $\mathrm{sh}_{\mathrm{t}}$ and $\mathrm{ax}_{\mathrm{b}}$. Plants resulting from suckers were used as control. Results. Parameters measured on the plants resulting from $\mathrm{ax}_{\mathrm{b}}$ did not present significant differences at the end of the acclimatization phase compared with the plants from $\mathrm{sh}_{\mathrm{t}}$, except for the foliar ratio. In the same way, parameters of vegetative growth in the field and those related to the yield were not significantly different between the two types of plants, $\mathrm{ax}_{\mathrm{b}}$ and $\mathrm{sh}_{\mathrm{t}}$. The reversion percentages of the type "false horn" towards the type "french" were $0.6 \%$ for the $\mathrm{sh}_{\mathrm{t}}$ plants and $1.2 \%$ for the $\mathrm{ax}_{\mathrm{b}}$ plants. Discussion and conclusion. Plants resulting from $s_{t}$ and $a_{b}$ behaved in the same manner during the acclimatization phase and in the field. The use of $\mathrm{ax}_{\mathrm{b}}$ makes it possible to increase the potentialities of in vitro production of healthy vegetable material. The reversion rate of the plants given by $a x_{b}$ was weak. The $a_{b}$ can thus be used like explants for the micropropagation of bananas. The same experiments on other cultivars pertaining to other genomic groups will make it possible to confirm or cancel these results.

Cameroon / Musa (plantains) / micropropagation / explants / propagation materials / growth / plant developmental stages / vegetative period / yields

Comportement de vitroplants de bananiers plantains issus de bourgeons axillaires et apicaux au cours de l'acclimatation et en champ.

Résumé - introduction. Le bananier plantain contribue à la sécurité alimentaire et aux revenus des populations de l'Afrique sub-saharienne. La qualité sanitaire du matériel végétal utilisé en bananeraies est un gage de leur pérennisation. Pour lever cette contrainte, les techniques de multiplication rapide du matériel végétal utilisent des méthodes de multiplication horticole in vivo ou de micropropagation in vitro. La micropropagation par bourgeonnement in vitro, qui permet une production massive de plants de bonne qualité sanitaire, ne fait appel habituellement qu'au bourgeon apical $\left(b_{a p}\right)$ ou terminal du rejet, alors que celui-ci possède également des bourgeons axillaires $\left(\mathrm{b}_{\mathrm{ax}}\right)$ dont nous avons évalué les potentialités. Matériel et méthodes. Nos expérimentations ont porté sur le cultivar Big Ebanga (groupe génomique AAB, sous-groupe des plantains, type fauxcorne). Nous avons comparé, au cours de l'acclimatation en ombrière et en champ, les paramètres de croissance et de développement de vitroplants issus des deux types d'explants $b_{a x}$ et $b_{a p}$. Des plants issus de rejets ont été utilisés comme témoins. Résultats. Les paramètres mesurés sur les plants issus de $b_{a x}$ n'ont pas présenté de différences significatives à la fin de l'acclimatation par rapport aux plants issus des $b_{a p}$, hormis le rapport foliaire. De même, les paramètres de croissance végétative en champ et ceux liés au rendement n'ont pas été significativement différents entre les deux types de plants $b_{a x}$ et $b_{a p}$. Les pourcentages de réversion du type " faux corne "vers le type "french " ont été de $0,6 \%$ pour les plants $b_{a p}$ et $1,2 \%$ pour plants $b_{a x}$. Discussion et conclusion. Les plants issus de $b_{a p}$ et $b_{a x}$ se sont comportés de la même manière pendant l'acclimatation en ombrière et en champ. L'utilisation de $\mathrm{b}_{\mathrm{ax}}$ permet d'augmenter les potentialités de production in vitro d'un matériel végétal de qualité. Le taux de réversion des plants $\mathrm{b}_{\mathrm{ax}}$ a été faible. Les $\mathrm{b}_{\mathrm{ax}}$ peuvent donc être utilisés comme explants pour la micropropagation du bananier. Des expérimentations effectuées sur d'autres cultivars appartenant à d'autres groupes génomiques permettront de confirmer ou infirmer ces résultats.

Cameroun / Musa (plantains) / micropropagation / explant / matériau de multiplication / croissance / stade de développement végétal / période végétative / rendement 


\section{Introduction}

Les bananiers sont cultivés dans plus de 100 pays des régions tropicales et subtropicales d'Afrique, d'Amérique et d'Asie [1]. Dans la plupart des pays, ils comptent parmi les cultures vivrières les plus importantes et jouent un rôle essentiel dans l'alimentation quotidienne. La banane est la quatrième denrée de base après le riz, le blé et les produits laitiers [2]. Sur les $88 \mathrm{Mt}$ produites annuellement dans le monde, à peine $10 \%$ sont destinées à l'exportation, l'essentiel étant consommé sur les lieux mêmes de la production. L'Afrique avec une production supérieure à 28 Mt totalise plus du tiers de la production mondiale de bananes, contribuant ainsi de façon significative à la sécurité alimentaire des populations. En raison de l'augmentation de la demande sur les marchés, régionaux et internationaux, les bananiers occupent une place prépondérante en tant que culture de rente, représentant dans certains cas l'unique source de revenus pour les populations rurales [3, 4]. Les bananiers constituent auss une importante source de fibres. Dans certains pays, les bananes permettent également de produire de l'alcool après fermentation (Burundi, Ouganda, Rwanda).

Les plantains constituent un sous-groupe du groupe génomique $\mathrm{AAB}$ composé de nombreux cultivars qui diffèrent les uns des autres par leur forme, leur taille, la couleur de leur pseudo-tronc, la taille et le poids du régime, la grosseur des doigts et le goût des fruits. Ces cultures étant conduites le plus souvent de manière extensive en association avec d'autres productions telles que le cacao, le café, l'arachide ou le haricot, leur rendement n'est généralement pas très élevé ( $5 \mathrm{t} \cdot \mathrm{ha}^{-1}$ environ) [5]. Les productions sont essentiellement concentrées en Amérique latine, dans les Caraïbes, en Afrique centrale et de l'Ouest.

L'augmentation de la productivité des plantations ne nécessite pas obligatoirement un accroissement des surfaces mais une conduite optimale de la parcelle mise en place sur un sol sain ou assaini avec un matériel végétal d'excellente qualité. Les différentes méthodes de propagation (multiplication sur souche décortiquée et plants issus des fragments de tige) ne satisfont pas la demande sans cesse croissante et le succès d'une plantation est donc étroitement lié à la qualité sanitaire du matériel végétal utilisé. Les différentes méthodes traditionnelles de multiplication au champ du matériel végétal (buttage, fausse décapitation, décapitation, pliage de la pseudo-tige) permettent une augmentation sensible du nombre de rejets, mais la qualité sanitaire de ce matériel est souvent médiocre.

La micropropagation par bourgeonnement in vitro, technique utilisée pour la production massive de vitroplants tant pour les bananiers de consommation locale que pour les plantations industrielles, n'utilise qu'un seul explant par rejet à savoir le bourgeon apical. Le rejet possède cependant des bourgeons axillaires qui peuvent être prélevés et micropropagés pour régénérer des vitroplants. Dans un travail récent, il a été montré que les bourgeons axillaires prolifèrent in vitro [6].

Le présent travail consiste à comparer les paramètres de croissance, le taux de réussite et la conformité de vitroplants issus de bourgeons axillaires ou de bourgeons apicaux, au cours de l'acclimatation et en champ.

\section{Matériel et méthodes}

\subsection{Matériel végétal}

La variété Big Ebanga, qui appartient au groupe génomique $\mathrm{AAB}$ (sous-groupe des plantains, type faux-corne), très appréciée au Cameroun, a servi de matériel végétal pour notre étude. Elle présente un bon potentiel de production de rejets, un cycle relativement court et de gros fruits de bonnes qualités gustatives. Deux types d'explants, issus de bourgeons apicaux et axillaires prélevés sur des rejets de cette variété provenant de la collection générale du Centre africain de recherches sur bananiers et plantains (Carbap, Cameroun), sont à l'origine des vitroplants que nous avons étudiés [7, 8]. Des rejets baïonnettes de cette variété ont été utilisés comme témoins en champ.

\subsection{Acclimatation}

L'acclimatation des vitroplants comprend deux étapes :

- le sevrage qui est l'adaptation progressive des vitroplants aux conditions extérieures 
(lumière, atmosphère, substrat) ; cette phase se prolonge jusqu'au stade où la dernière feuille mesure au moins $9 \mathrm{~cm}$ de long ;

- l'élevage qui consiste à faire croître la plante jusqu'au stade où la dernière feuille a atteint une longueur de $25 \mathrm{~cm}$.

Le substrat utilisé au cours de ces deux étapes a été composé d'un mélange de terre volcanique et de parche de café dans les proportions 1:1(v/v). Le substrat homogène a été désinfecté par chauffage sur feu de bois dans des fûts de $200 \mathrm{~L}$ pendant $12 \mathrm{~h}$.

Pendant l'acclimatation, les vitroplants issus des bourgeons axillaires et apicaux ont été disposés en blocs complètement randomisés à cinq répétitions. Chaque répétition ou parcelle élémentaire a été constituée de 60 vitroplants : 30 issus de bourgeons axillaires et 30 de bourgeons apicaux.

\subsubsection{Sevrage (serre)}

Pour le sevrage des vitroplants, le substrat a été tassé dans des conteneurs de $125 \mathrm{~mL}$ à fond perforé permettant un bon drainage. Après arrosage, ils ont été disposés sur des plateaux d'une capacité maximale de 90 pots.

En fin de phase de croissance, les vitroplants ont été isolés, nettoyés à l'eau du robinet et rincés dans une solution de benlate $\left(0,5 \mathrm{~g} \cdot \mathrm{L}^{-1}\right)$ avant d'être repiqués. Le repiquage a été effectué à l'ombre et les plants repiqués ont rapidement été transférés sous tunnel. Quatre brumisations de $1 \mathrm{~min}$ chacune ont été réalisées chaque jour.

La fertilisation a été effectuée par brumisation manuelle, en fin de journée, avec une solution de Fertigofol 313 à $8 \mathrm{~mL} \cdot \mathrm{L}^{-1}$. Le sevrage des plants a duré 28 jours. Tous les plants ont été transférés sous ombrière lorsque $70 \%$ des plants repiqués présentaient des feuilles de la longueur requise $(9 \mathrm{~cm})$.

\subsection{2. Élevage (ombrière)}

Pour l'élevage des plantules sevrées, le substrat de culture a été tassé dans des sachets perforés de 2,5 L en polyéthylène noir ; ils ont été ensuite disposés sous ombrière. Le substrat a été arrosé abondamment puis laissé à décanter pendant quelques heures ou jours avant le repiquage.

Les plantules ont été repiquées dans les sachets avec leur motte. L'apport d'eau a été fait par aspersion à raison d'un arrosage par jour.

Un apport hebdomadaire d'engrais foliaire a été fait par pulvérisation de Fertigofol 313 à la même concentration que précédemment. Une fertilisation au NPK 20.10.10 $\left(2,5 \mathrm{~g} \cdot\right.$ plant $^{-1} \cdot$ semaine $\left.^{-1}\right)$ a été réalisée à partir de la deuxième semaine après repiquage jusqu'à la fin de l'élevage. Cette phase a duré 42 jours et $70 \%$ des plants ont présenté alors des dernières feuilles ayant la taille optimale $(25 \mathrm{~cm})$ pour être transférés au champ.

\subsection{Conduite des plants en champ}

Une parcelle préalablement mise en jachère a été nettoyée ; un piquetage $(3 \mathrm{~m} \times 2 \mathrm{~m})$ a été effectué, ainsi qu'une trouaison ( $40 \mathrm{~cm} \times$ $40 \mathrm{~cm}$ ). Les vitroplants issus des bourgeons axillaires et apicaux ainsi que les rejets ont été mis en champ le 24 juillet 2000. La parcelle a été conduite pendant trois cycles de culture. Chaque plant a reçu 35 g d'urée, 10 fois par an ; $100 \mathrm{~g}$ de sulfate d'ammonium et $100 \mathrm{~g}$ de potasse tous les 6 mois, mais également $20 \mathrm{~g}$ de Régent (insecticide) et $20 \mathrm{~g}$ de Rugby (nématicide) tous les 4 mois. Le traitement contre la maladie des raies noires a été réalisé mensuellement avec des benzimidazoles ou des triazoles. Le désherbage et l'irrigation en saison sèche ont été faits de façon systématique.

Pendant la conduite des plants en champ, les vitroplants issus de bourgeons axillaires et apicaux, de même que les rejets, ont été disposés en blocs complètement randomisés à 5 répétitions. Une répétition ou parcelle élémentaire a consisté en 90 plants, soit 30 plants de chaque type.

\subsection{Caractéristiques des plants}

\subsubsection{Paramètres mesurés au cours de l'acclimatation}

Des observations ont été réalisées toutes les semaines après 2 semaines d'adaptation des plantes dans chacune des deux phases de sevrage et d'élevage. Deux types de paramètres biologiques ont été étudiés :

- des paramètres permettant d'évaluer la croissance des plants : hauteur, diamètre de 
la pseudo-tige, longueur et largeur des feuilles ;

- des caractéristiques rendant compte de leur développement : nombre de feuilles émises, rythme d'émission foliaire hebdomadaire (nombre de feuilles émises par semaine), surface foliaire (Sf) telle que [Sf = longueur de la feuille $\times$ largeur de la feuille $\times 0,83][9,10]$ et rapport foliaire $(\mathrm{kf})$ tel que $[\mathrm{kf}=$ longueur de la feuille/largeur de la feuille] [9, 10].

Le taux de réussite par type d'explant a été évalué. L'homogénéité des plants a été contrôlée.

\subsubsection{Paramètres mesurés au cours de la phase végétative au champ}

Au champ, les caractéristiques de croissance étudiées ont été la hauteur des plants et le diamètre de la pseudo-tige à $10 \mathrm{~cm}$ du sol.

Les caractéristiques du développement observées ont été le nombre de feuilles émises, le nombre de feuilles vivantes, le nombre de rejets formés mesurant plus de $1 \mathrm{~m}$ de hauteur et le nombre de rejets formés inférieurs à $1 \mathrm{~m}$.

\subsubsection{Paramètres mesurés en phase de production}

Les observations effectuées sur la phase de production ont porté sur l'intervalle plantation-floraison, l'intervalle floraison-coupe, le poids du régime récolté, le nombre de mains du régime, le nombre de doigts par main, la longueur du doigt médian, le grade du doigt médian de chaque main, le poids moyen des mains du régime et le poids moyen des doigts médians.

\subsection{4. Étude de la conformité}

L'étude de la conformité a été effectuée à partir de l'observation de la couleur, la forme et la taille des feuilles des vitroplants, puis de la conformité du régime, la forme du fruit et le nombre de mains par régime.

\subsubsection{Analyse statistique}

Les données ont été analysées à l'aide du logiciel SAS et la comparaison des moyennes a été faite par le test de Student. Le seuil de $5 \%$ a été retenu comme seuil de signification.

\section{Résultats}

\subsection{Caractéristiques des vitroplants de différentes origines au cours de l'acclimatation}

\subsubsection{Diamètre de la pseudo-tige}

Les diamètres moyens de la pseudo-tige des plants issus de bourgeons axillaires $\left(\mathrm{b}_{\mathrm{ax}}\right)$ et de ceux issus de bourgeons apicaux $\left(b_{a p}\right)$ ont été significativement différents à la fin de la phase de sevrage $(5,65 \mathrm{~mm}$ pour les vitroplants issus de $b_{a x}, 5,81 \mathrm{~mm}$ pour les bourgeons issus de $b_{a p}$ ), mais non significativement différents à la fin de la phase d'élevage $\left(14,28 \mathrm{~mm}\right.$ pour $\mathrm{b}_{\mathrm{ax}}, 14,40 \mathrm{~mm}$ pour $\left.\mathrm{b}_{\mathrm{ap}}\right)$ (tableau I).

\subsubsection{Hauteur de la pseudo-tige}

À la fin de la phase de sevrage, les hauteurs moyennes de la pseudo-tige des plants issus des bourgeons apicaux $(5,85 \mathrm{~cm})$ et de ceux issus de bourgeons axillaires $(5,58 \mathrm{~cm})$ ont été significativement différentes. Toutefois, elles n'ont pas été significativement différentes à la fin de la phase d'élevage (tableau I).

\subsubsection{Surface foliaire}

À la fin de la phase de sevrage, les différences observées entre la surface moyenne des plants issus de bourgeons apicaux $\left(16,28 \mathrm{~cm}^{2}\right)$ et celle des plants issus de bourgeons axillaires $\left(11,83 \mathrm{~cm}^{2}\right)$ ont été très significatives. À la fin de la phase de l'élevage, les surfaces moyennes respectives ont été de $279,50 \mathrm{~cm}^{2}$ et de $283,55 \mathrm{~cm}^{2}$ pour les bourgeons apicaux et axillaires (tableau I).

\subsubsection{Rythme d'émission foliaire (nombre de feuilles émises par semaine)}

L'analyse des courbes présentant l'influence du type de bourgeon à l'origine du vitroplant placé en phase d'acclimatation sur son rythme d'émission foliaire a mis en évidence deux phases (figure 1):

- une phase d'émission lente et irrégulière s'étendant du $1^{\mathrm{er}}$ au $49^{\mathrm{e}}$ jour après repiquage pendant laquelle le nombre de feuilles émises est inférieur à 1 ; 


\begin{tabular}{|c|c|c|c|c|c|c|c|}
\hline \multirow[t]{2}{*}{ Acclimatation } & \multirow[t]{2}{*}{$\begin{array}{l}\text { Temps } \\
\text { (jours) }\end{array}$} & \multicolumn{2}{|c|}{$\begin{array}{l}\text { Hauteur de la pseudo-tige } \\
\qquad(\mathrm{cm})\end{array}$} & \multicolumn{2}{|c|}{$\begin{array}{l}\text { Diamètre des pseudo-troncs } \\
\qquad(\mathrm{mm})\end{array}$} & \multicolumn{2}{|c|}{$\begin{array}{l}\text { Surface foliaire des vitroplants } \\
\qquad\left(\mathrm{cm}^{2}\right)\end{array}$} \\
\hline & & $\begin{array}{l}\text { Issus de } \\
\text { bourgeons } \\
\text { apicaux }\end{array}$ & $\begin{array}{l}\text { Issus de } \\
\text { bourgeons } \\
\text { axillaires }\end{array}$ & $\begin{array}{l}\text { Issus de } \\
\text { bourgeons } \\
\text { apicaux }\end{array}$ & $\begin{array}{l}\text { Issus de } \\
\text { bourgeons } \\
\text { axillaires }\end{array}$ & $\begin{array}{l}\text { Issus de } \\
\text { bourgeons } \\
\text { apicaux }\end{array}$ & $\begin{array}{l}\text { Issus de } \\
\text { bourgeons } \\
\text { axillaires }\end{array}$ \\
\hline \multirow{5}{*}{$\begin{array}{l}\text { Phase de } \\
\text { sevrage }\end{array}$} & 0 & $3,38 \pm 0,75$ & $3,62 \pm 0,71$ & $4,80 \pm 0,62$ & $5,12 \pm 0,64$ & - & - \\
\hline & 14 & $4,12 \pm 0,78$ & $4,33 \pm 0,72$ & $5,82 \pm 0,76$ & $5,86 \pm 0,90$ & $12,90 \pm 5,92$ & $11,59 \pm 5,42$ \\
\hline & 21 & $4,89 \pm 1,03$ & $4,89 \pm 0,94$ & $5,84 \pm 0,93$ & $5,73 \pm 0,87$ & $11,08 \pm 10,19$ & $10,07 \pm 8,85$ \\
\hline & 28 & $5,85 \pm 1,15$ & $5,58 \pm 1,11$ & $5,81 \pm 0,91$ & $5,65 \pm 0,90$ & $16,28 \pm 1,13$ & $11,83 \pm 11,18$ \\
\hline & $p$ & \multicolumn{2}{|c|}{$<0,05$} & \multicolumn{2}{|c|}{$>0,05$} & \multicolumn{2}{|c|}{$<0,05$} \\
\hline \multirow{6}{*}{$\begin{array}{l}\text { Phase } \\
\text { d'élevage }\end{array}$} & 42 & $6,05 \pm 1,14$ & $5,62 \pm 1,02$ & $6,48 \pm 1,14$ & $5,81 \pm 0,86$ & $26,83 \pm 10,98$ & $22,66 \pm 8,96$ \\
\hline & 49 & $6,87 \pm 1,20$ & $6,98 \pm 1,20$ & $7,20 \pm 1,28$ & $7,13 \pm 1,30$ & $35,49 \pm 13,08$ & $39,73 \pm 15,64$ \\
\hline & 56 & $8,43 \pm 1,45$ & $8,16 \pm 1,54$ & $8,28 \pm 1,50$ & $8,51 \pm 1,37$ & $73,79 \pm 31,55$ & $74,44 \pm 31,83$ \\
\hline & 63 & $10,82 \pm 1,99$ & $10,78 \pm 2,05$ & $10,92 \pm 1,76$ & $11,00 \pm 1,64$ & $155,46 \pm 64,83$ & $144,21 \pm 63,62$ \\
\hline & 70 & $15,03 \pm 2,41$ & $15,04 \pm 2,42$ & $14,40 \pm 2,36$ & $14,28 \pm 2,42$ & $276,50 \pm 89,86$ & $283,55 \pm 94,51$ \\
\hline & $p$ & \multicolumn{2}{|c|}{$>0,05$} & \multicolumn{2}{|c|}{$>0,05$} & \multicolumn{2}{|c|}{$>0,05$} \\
\hline
\end{tabular}

- une phase d'émission régulière et stable s'étendant du $50^{\mathrm{e}}$ au $70^{\mathrm{e}}$ jour après repiquage pendant laquelle le nombre de feuilles émises est supérieur à 1 .

En fin de phases de sevrage et d'élevage, la différence observée entre les deux types de vitroplants n'a pas été significative. Le nombre de feuilles émises moyen a été, en fin du sevrage, de 0,70 pour les vitroplants issus de bourgeons apicaux et de 0,60 pour ceux issus de bourgeons axillaires et, en fin d'élevage de 1,14 pour les vitroplants issus de bourgeons apicaux et 1,21 pour ceux issus de bourgeons axillaires.

L'intervalle de temps qui s'écoule entre l'émission de deux feuilles consécutives ou phyllochrone a varié de (5 à 14) jours au cours du sevrage. En phase d'élevage, pendant les 21 premiers jours, il a varié de $(5$ à 11) jours. Après cette phase d'adaptation à l'élevage, il s'est situé entre (4 et 6) jours.

\subsubsection{Rapport foliaire}

Pour les deux types de vitroplants étudiés, l'évolution du rapport foliaire $\mathrm{kf}$ [= longueur de la feuille/largeur de la feuille] a présenté des fluctuations importantes (figure 2) carac-

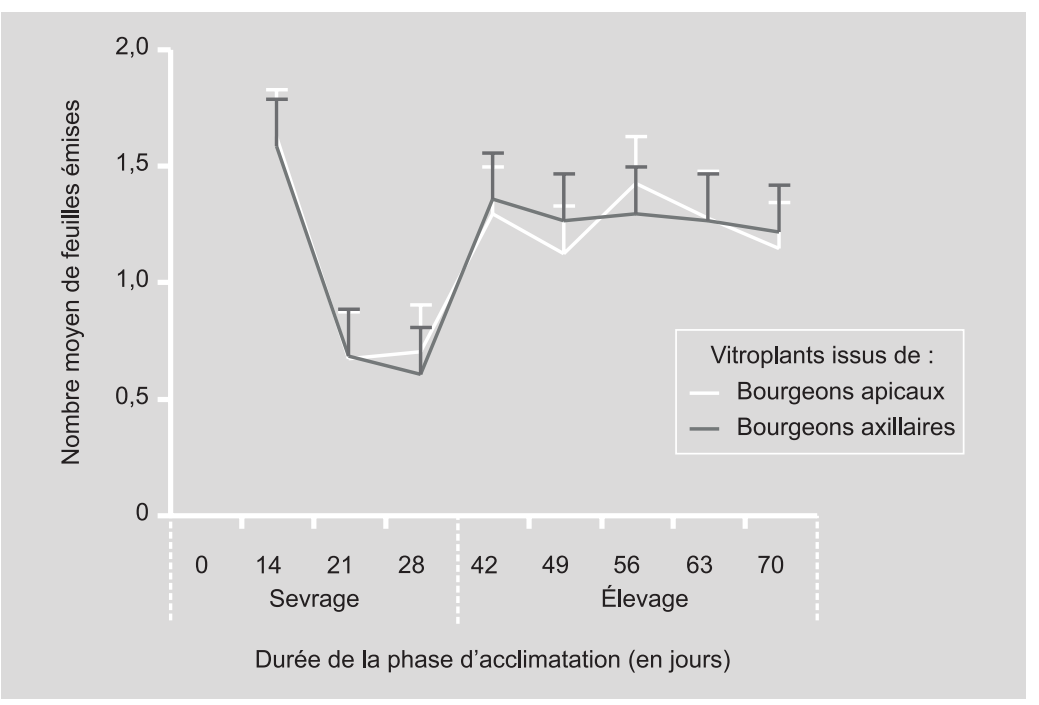

Figure 1.

Évolution du rythme d'émission foliaire moyen (ou nombre moyen de feuilles émises par semaine) de vitroplants de bananiers issus de la micropropagation de deux types de bourgeons pendant leur phase d'acclimatation.

térisées par une phase de ralentissement et la chute de cette valeur entre le début du sevrage et le $21^{\mathrm{e}}$ jour, suivie d'une phase 


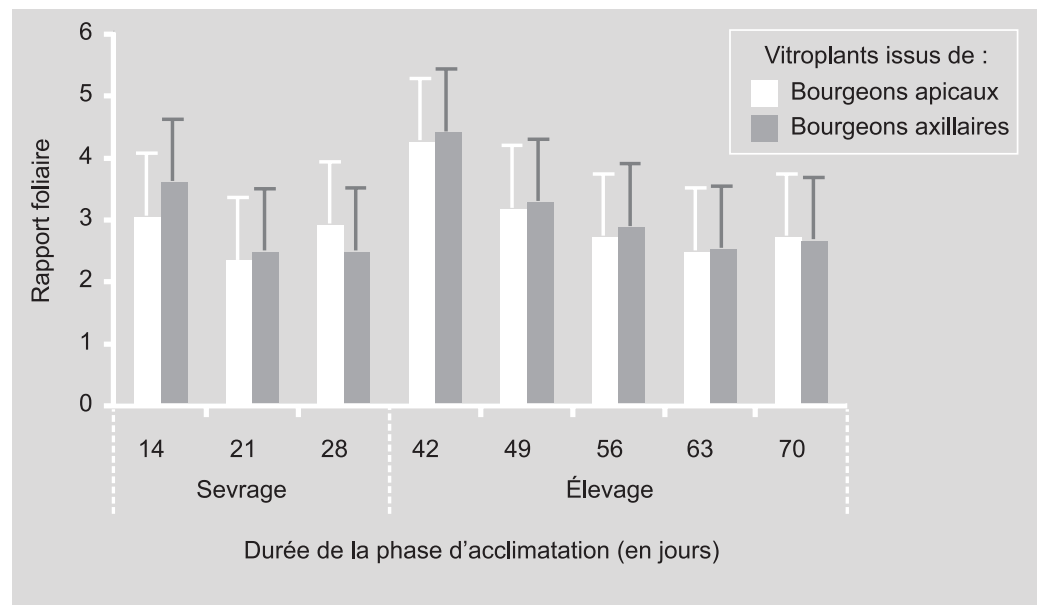

Figure 2.

Évolution du rapport foliaire de vitroplants de bananiers issus de la micropropagation de deux types de bourgeons pendant leur phase d'acclimatation.

\subsubsection{En champ}

Les observations faites en champ ont montré que les plants issus de bourgeons axillaires présentaient des réversions du type faux corne vers le type french avec un pourcentage de $1,2 \%$ dès le premier cycle, alors que ceux issus de bourgeons apicaux présentaient un taux de réversion plus faible de $0 \%$ au premier cycle et de $0,6 \%$ au deuxième cycle. Au troisième cycle, $2 \%$ de plants issus des bourgeons axillaires ont présenté des régimes anormaux avec les trois premières mains conformes. Seuls les témoins sont restés homogènes au cours des trois cycles (tableau II).

\subsection{Comparaison des bananiers issus de vitroplants et de ceux issus de rejets (témoins)}

Les principales caractéristiques agronomiques des différents types de bananiers obtenus soit de vitroplants soit de rejets traditionnels ont été comparées (tableau III).

$\mathrm{Au}$ premier et troisième cycle, la hauteur des plants issus des bourgeons apicaux s'est révélée supérieure à celle des plants issus des bourgeons axillaires et des témoins ; cette différence n'a pas été observée au second cycle.

À tous les cycles, la circonférence du pseudo-tronc à $10 \mathrm{~cm}$ du sol a été identique chez les plants issus des deux types de bourgeons. Cette valeur a été inférieure chez les bananiers issus des rejets.

Pour les trois cycles observés, le nombre de feuilles émises et le nombre de feuilles vivantes, identique chez les vitroplants, a été relativement élevé, alors que ces mêmes valeurs restaient plus faibles chez les plants issus de rejets. Le rejetonnage a été dans l'ensemble inférieur pour les bananiers issus de rejets.

Le poids moyen du régime a été plus important chez les vitroplants (11,9 kg, en moyenne au troisième cycle) alors qu'il a été de $9,3 \mathrm{~kg}$ chez les rejets. Il en a été de même pour le nombre de mains et de doigts par régime, identique chez les vitroplants avec, cependant, une légère dominance au premier cycle chez les plants issus de bourgeons axillaires, et un nombre de mains et 
Tableau II.

Étude de la conformité des plants de bananiers de différentes origines (vitroplants issus de bourgeons apicaux ou axillaires ou plants obtenus de rejets baïonnettes) pendant les phases végétatives et fructifères, au cours de trois cycles d'observation.

\begin{tabular}{|c|c|c|c|c|c|c|}
\hline $\begin{array}{l}\text { Cycle } \\
\text { d'observation }\end{array}$ & Origine du vitroplant & $\begin{array}{l}\text { Nombre de variants } \\
\text { Sur } 150 \text { plants }\end{array}$ & Formes des feuilles & Type de régime & Type de fruit & $\begin{array}{c}\text { Nombre de mains } \\
\text { par régime }\end{array}$ \\
\hline \multirow[t]{3}{*}{$1^{\mathrm{er}}$ cycle } & Bourgeon apical & 0 & Normales & Normal & Normal & Normal \\
\hline & Bourgeon axillaire & 2 & $\begin{array}{l}\text { Panachées, épaisses, } \\
\text { effilées, rayures }\end{array}$ & Non conforme & French clair & 7 \\
\hline & Rejet & 0 & - & Normal & Normal & Normal \\
\hline \multirow[t]{3}{*}{$2^{\mathrm{e}}$ cycle } & Bourgeon apical & 1 & Effilées & Non conforme & French clair & 7 \\
\hline & Bourgeon axillaire & 2 & $\begin{array}{c}\text { Panachées, épaisses, } \\
\text { effilées, rayures }\end{array}$ & Non conforme & French clair & 7 \\
\hline & Rejet & 0 & - & Normal & Normal & Normal \\
\hline \multirow[t]{4}{*}{$3^{\mathrm{e}}$ cycle } & Bourgeon apical & 1 & Effilées & Non conforme & French clair & 7 \\
\hline & Bourgeon axillaire & 2 & $\begin{array}{l}\text { Panachées, épaisses, } \\
\text { effilées, rayures }\end{array}$ & Non conforme & French clair & 7 \\
\hline & & 3 & normales & Non conforme & Big Ebanga & 3 \\
\hline & Rejet & 0 & Normales & Normal & Normal & Normal \\
\hline
\end{tabular}

de doigts par régime faible chez les bananiers issus de rejets.

Si la longueur des doigts des régimes des plants provenant des vitroplants a présenté des différences significatives au premier cycle, elle a été identique chez les trois types de plants au second cycle et une légère domination de cette valeur a pu être notée chez les vitroplants en troisième cycle.

Le grade a été plus élevé au premier cycle chez les plants issus de bourgeons axillaires et de rejets que chez les plants issus de bourgeons apicaux, mais cette valeur n'a pas présenté de différences significatives au second cycle. Au troisième cycle, les plants issus des bourgeons axillaires ont eu des grades identiques et supérieurs à ceux observés chez les plants issus de rejets.

Pour les trois types de plants, le poids moyen des mains et le poids moyen du doigt médian n'ont varié ni au premier, ni au second cycle, mais, pour les bananiers obtenus de rejets, ces valeurs ont diminué au troisième cycle.

Au premier et au second cycle, la floraison a été précoce chez les plants issus de bourgeons axillaires, mais, au troisième cycle, ces différences n'ont plus été significatives. Par rapport aux régimes produits par les plants obtenus de vitroplants, les régimes issus des rejets ont mûri tardivement au premier cycle, tandis qu'au second et au troisième cycle, les trois types de plants ont donné des régimes qui ont mûri dans le même intervalle de temps (68 à 80 jours).

En fin de troisième cycle, les bananiers obtenus de vitroplants issus des bourgeons axillaires et des bourgeons apicaux se sont comportés de la même façon pour les paramètres étudiés ; le poids des régimes produits par les plants issus de rejets a été dans l'ensemble d'un poids inférieur à celui des bananiers issus de vitroplants.

\section{Discussion}

Le bon comportement des plants issus de bourgeons axillaires en fin de phase de croissance in vitro traduit une aptitude à la prolifération qui pourrait être expliquée par une faible dominance apicale.

Lorsque les bourgeons axillaires sont séparés du pied mère, ils sont isolés des 


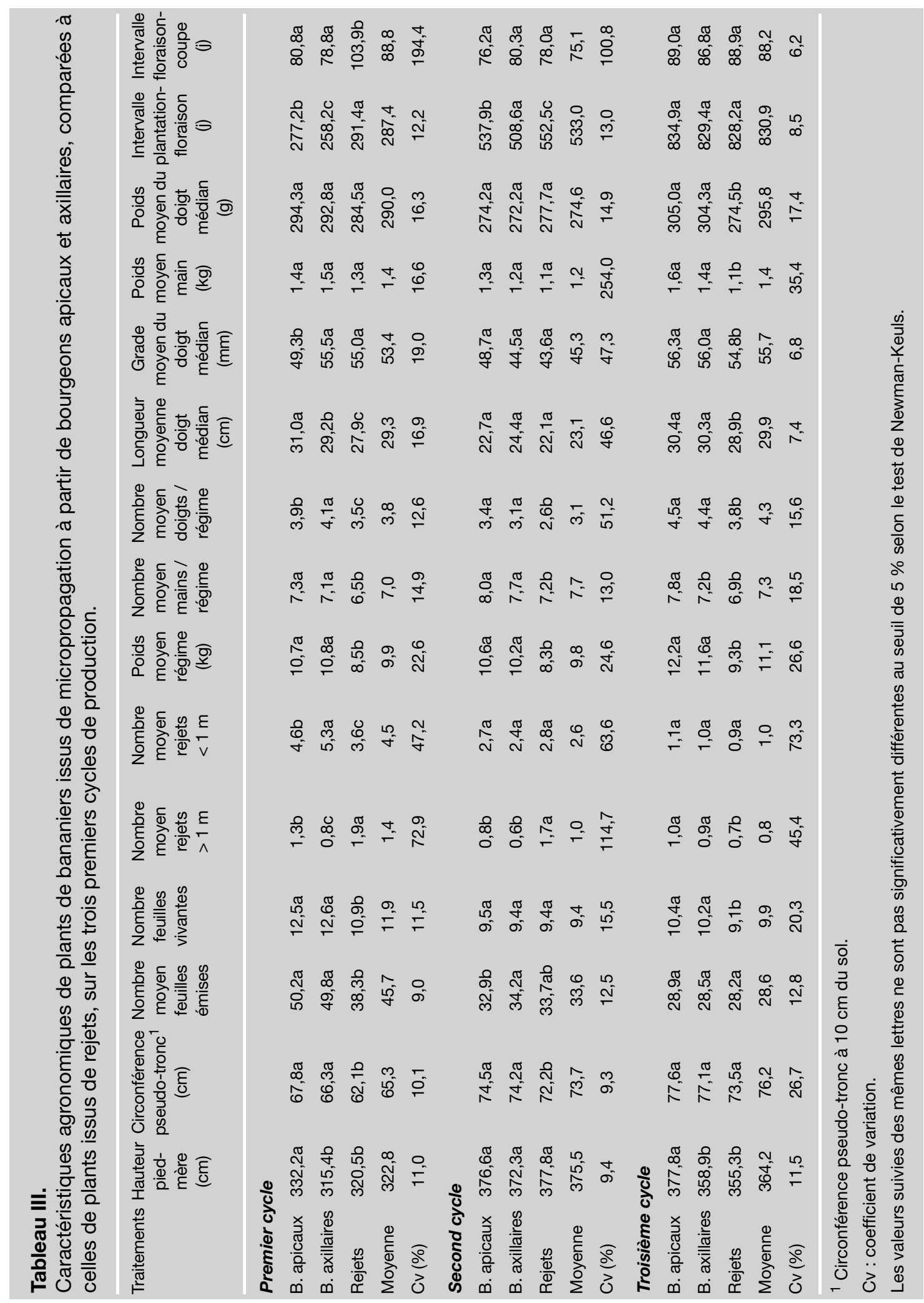


corrélations morphogénétiques. La levée précoce des bourgeons axillaires permet un démarquage des plants qui en dérivent dans le mouvement morphogénétique. Sur la tige du bananier, il existe deux champs morphogénétiques qui seraient sollicités pour la formation des bourgeons : les champs internodal et nodal [11]. Pendant la vie d'une plante, ces champs peuvent être latents ou actifs. Les différences observées sur un même type d'explant ou sur des types d'explants distincts seraient liées au site de formation du bourgeon d'une part, à l'exploitation variable de leurs potentialités morphogénétiques en culture in vitro, plus précisément à l'AGMA (Activité Globale du Méristème Apical) ou à l'absence de synchronisation de ce processus, d'autre part [11]. Les différentes phases de croissance observées confirment l'influence des facteurs externes sur l'acclimatation des vitroplants [8, 12, 13].

L'étude des caractéristiques agronomiques a montré des similitudes entre les plants issus des bourgeons axillaires et ceux issus des bourgeons apicaux. En effet, si, au premier cycle, les plants issus des bourgeons axillaires ont été précoces, ont rejetonné davantage et ont présenté un nombre de doigts plus élevé avec un grade important, ceux issus des bourgeons apicaux ont présenté plus de rejets supérieurs à $100 \mathrm{~cm}$, la longueur de leurs doigts médians a été plus élevée et l'intervalle plantation-récole a été plus long. Ces différences n'ont plus été significatives au second et au troisième cycle. Cela permet d'affirmer que les plants issus des bourgeons axillaires présentent les mêmes caractéristiques agronomiques que ceux issus des bourgeons apicaux.

Le pourcentage de mutation ou de réversion classique chez les plantains (de faux corne en french) a été de 0,6\% chez les plants issus de bourgeons apicaux alors qu'il a été de 1,2\% chez ceux issus de bourgeons axillaires. Chez les plants issus de bourgeons axillaires, 0,18\% de plants ont présenté des régimes avec un nombre de mains réduit au troisième cycle. Ce pourcentage reste encore très faible par rapport aux observations antérieures faites chez ce même cultivar qui faisaient état d'un taux de variation de 25,6 [14, 15].

\section{Conclusion}

Les vitroplants issus de bourgeons axillaires ont présenté, au cours de l'acclimatation, une croissance et un développement similaires aux plants issus des bourgeons apicaux. Leur taux de reprise (100\%) et l'absence de variants observés au cours des phases de sevrage et d'élevage font des bourgeons axillaires des explants potentiels pour la micropropagation du bananier. Les observations faites sur les caractéristiques agronomiques en champ n'ont pas montré de différences significatives aux second et troisième cycles. Les bananiers issus de vitroplants ont tous été plus performants que les rejets (témoins). Des cas isolés de réversion ont été relevés chez de tels plants, mais avec un faible pourcentage. Ces résultats permettent d'envisager l'utilisation des bourgeons axillaires comme explants de départ pour la micropropagation du bananier plantain. Chez d'autres variétés et groupes génomiques de bananiers, il faudra préalablement tester ce type de matériel avant de généraliser ou non l'utilisation de tels explants.

\section{Références}

[1] Bakry F., Careel F., Caruana M.L., Côte F.X., Jenny C., Tézénas du Montcel H., Les bananiers, in: L'amélioration des plantes tropicales, collection Repères, Cirad-Orstom, Montpellier, France,1997, 109-139.

[2] Scoofs H., Panis B., Stosse H., Mayo Mosqueda A., Lopez Torres J., Roux N., Dolezel J., Swennen R., Difficultés rencontrées pour établir et maintenir des suspensions cellulaires morphogènes de bananier et pour régénérer les plants par embryogenèse somatique, Infomusa 8 (1998) 21-35.

[3] Frison E., Sharrock S., Les productions bananières : un enjeu économique majeur pour la sécurité alimentaire, in: Bananas and food security, Crbp-Cta-Inibap, Montpellier, France, 1998, 21-35.

[4] Temple L., Quantification des productions et des échanges de fruits et légumes au Cameroun, Cah. Agric. 10 (2001) 87-94.

[5] Lescot T., Les bananiers, une diversité méconnue, Fruitrop 51 (1998) 9-12. 
E. Youmbi et al.

[6] Youmbi E., Ngaha D., Expression in vitro des capacités organogènes des bourgeons axillaires chez le bananier plantain (Musa spp.), Fruits 59 (4) (2004) 241-248.

[7] Israeli Y., Lahav E., Reuveni O., In vitro culture of bananas, in: Gowen S. (Ed.), Bananas and plantains, Chapman and Hall, World Crop Series, London, UK, 1995, 147-178.

[8] Vuylsteke D.R., Swennen R., De Langhe E., Shoot-tip culture for the propagation, conservation, and distribution of Musa germplasm, IITA, Ibadan, Nigeria, 1998.

[9] Ganry J., Action de la température et du rayonnement solaire sur la vitesse de croissance des feuilles du bananier (Musa acuminata Colla) - Application à l'étude du rythme et du développement de la plante et relation avec la productivité, Univ. Paris VII, thèse, Paris, France, 1980, 331 p.

[10] Saya A., Étude du comportement au sevrage et au champ des vitroplants endurcis : mise au point d'un protocole standard simplifié,
Rapport de stage post-doctoral, CRBP/ AUPELF, Cameroun, 1994, 27 p.

[11] Kwa M., Architecture, morphogenèse et anatomie de quelques cultivars de bananiers, Univ. Montpellier II, thèse, Montpellier, France, 1993, $286 \mathrm{p}$.

[12] Vuylsteke D., Talengera D., Postflask management of micropropagated bananas and plantains, IITA, Ibadan, Nigeria, 1998,15 p.

[13] Djaha J.B.A., N'Guessan A.E., Sevrage et élevage des vitroplants de bananiers : incidence du rayonnement solaire global sur la croissance et le développement des bananiers plantains, IRFA, Azaguié, Côte d'Ivoire, 1991, $14 \mathrm{p}$

[14] Vuylsteke D., Swennen R., De Lange E., Somaclonal variation in plantains (Musa spp., AAB group) derived from shoot-tip culture, Fruits 46 (1991) 429-439.

[15] Vuylsteke D., Oritz R., Ferris S., Crouch J., Plantain improvement, Plant Breeding 14 (1997) 267-320.

\section{Comportamiento in vitro de plátanos resultantes de yemas axilares y apicales durante la aclimatación y en campo.}

Resumen - introducción. El plátano contribuye a la seguridad alimenticia y a los ingresos de las poblaciones del África subsahariana. La calidad sanitaria del material vegetal utilizado en plantaciones es una prueba de su perennidad. Para suprimir esta traba, las técnicas de multiplicación rápida del material vegetal utilizan métodos de multiplicación hortícola in vivo o de micropropagación in vitro. La micropropagación mediante injertos in vitro, que permite una producción masiva de plantas de buena calidad sanitaria, sólo recurre habitualmente a la yema apical $\left(b_{a p}\right)$ o a la espiga floral del brote, mientras que éste posee además también yemas axilares $\left(b_{a x}\right)$ cuyas potencialidades evaluamos. Material y métodos. Nuestros experimentos se refirieron al cultivar Big Ebanga (grupo genómico AAB, subgrupo de los plátanos, tipo faux-corne). Comparamos, durante la aclimatación en sombrío y en campo, los parámetros de crecimiento $\mathrm{y}$ de desarrollo in vitro resultantes de los dos tipos de explantes $\mathrm{b}_{\mathrm{ax}}$ y $\mathrm{b}_{\mathrm{ap}}$. Se utilizaron algunas plantas resultantes de brotes como testigos. Resultados. Los parámetros medidos sobre las plantas resultantes de $b_{\mathrm{ax}}$ no presentaron diferencias significativas al final de la aclimatación con relación a las plantas resultantes de los $\mathrm{b}_{\mathrm{ap}}$, salvo en su relación foliar. Asimismo, los parámetros de crecimiento vegetativo en campo y aquellos vinculados al rendimiento no fueron significativamente diferentes entre los dos tipos de plantas $b_{a x}$ y $b_{a p}$. Los porcentajes de reversión del tipo "Faux-corne" hacia el tipo "French" fueron del 0,6\% para las plantas $b_{a p}$ y del1,2\% para plantas $\mathrm{b}_{\mathrm{ax}}$. Discusión y conclusión. Las plantas resultantes de $\mathrm{b}_{\mathrm{ap}} \mathrm{y} \mathrm{b}_{\mathrm{ax}}$ se comportaron de la misma manera durante la aclimatación en sombrío y en campo. La utilización de $\mathrm{b}_{\mathrm{ax}}$ permite aumentar las potencialidades de producción in vitro de un material vegetal de calidad. El porcentaje de reversión de las plantas $\mathrm{b}_{\mathrm{ax}}$ fue flojo. Los $\mathrm{b}_{\mathrm{ax}}$ pueden pues utilizarse como explantes para la micropropagación del plátano. Experimentos efectuados en otros cultivares que pertenezcan a otros grupos genómicos permitirán confirmar o invalidar estos resultados.

Camerún / Musa (plátanos) / micropropagación / explantes / materiales de propagación / crecimiento I etapas de desarrollo de la planta I periodo vegetativo / rendimiento 